\title{
Erratum to: Association of allelic variation in two NPRI-like genes with Fusarium head blight resistance in wheat
}

\author{
Manuela Diethelm • Michael Schmolke • \\ Jennifer Groth • Wolfgang Friedt • \\ Günther Schweizer · Lorenz Hartl
}

Published online: 18 April 2014

(C) Springer Science+Business Media Dordrecht 2014

\section{Erratum to: Mol Breeding DOI 10.1007/s11032-013-0010-2}

We thank Dr. Anna Gordon from NIAB in Cambridge, $\mathrm{UK}$, for spotting discrepancies in marker designations in Fig. 1. We confounded designations of markers for TDF_076_2A and TDF_076_2D which affects five parts of the manuscript. The authors apologize to the readers of Molecular Breeding for all inconveniences caused by these mistakes. The paper should be modified as follows:

The online version of the original article can be found under doi:10.1007/s11032-013-0010-2.

M. Diethelm $(\varangle) \cdot$ J. Groth · G. Schweizer · L. Hartl Institute for Crop Science and Plant Breeding, Bavarian State Research Center for Agriculture, Am Gereuth 8, 85354 Freising, Germany

e-mail: manuela.diethelm@1fl.bayern.de

M. Schmolke

Plant Breeding, Technische Universität München,

Emil-Ramann-Str. 4, 85354 Freising, Germany

Present Address:

M. Schmolke

Bayer CropScience AG, European Wheat Breeding Center, Am Schwabeplan 6, OT Gatersleben, 06466 Stadt Seeland, Germany

W. Friedt

Plant Breeding Department, Justus Liebig University, Heinrich-Buff-Ring 26-32, 35392 Giessen, Germany
1. Under the side heading "Sequence analysis of TDF_076": The lines "Genetic mapping of these markers showed that TDF_076_INDEL1, TDF_ 076_INDEL2, TDF_076_SNP1 and TDF_076_ SNP12 $(\mathrm{T}=$ allele 2$)$ mapped to the long arm of chromosome 2A, while TDF_076_INDEL3, TDF_076_SNP13 and TDF_076_SNP12 (A = allele 2) mapped on the long arm of chromosome 2D (Fig. 1)" should read as "Genetic mapping of these markers showed that TDF_076_INDEL3, TDF_076_INDEL2, TDF_076_SNP1 and TDF_ 076_SNP12 ( $\mathrm{T}=$ allele 2) mapped to the long arm of chromosome 2A, while TDF_076_INDEL1 and TDF_076_SNP12 $(\mathrm{A}=$ allele 1$)$ mapped on the long arm of chromosome 2D (Fig. 1).”

2. The corrected Fig. 1 is given in this Erratum.

3. In Table 1, SNP13: the haplotype 1 of TDF_076_ 2D has $\mathrm{T}$ and haplotype 2 has $\mathrm{A}$.

4. Under the side heading "Effect of TDF_076, TDF_032 and TDF_102 in a diverse collection of European winter wheat cultivars": The lines "Genotypes with a combination of haplotype 1 of TDF_076_2A and haplotype 2 of TDF_076_2A had $14.2 \%$ less infected spikelets than genotypes with the negative allele 1 and $3 \%$ more infected spikelets than genotypes with the positive allele 2 of both homoeologous genes" should read as "Genotypes with a combination of haplotype 1 of TDF_076_2A and haplotype 2 of TDF_076_2D had $14.2 \%$ less infected spikelets than genotypes with the negative allele 1 and $3 \%$ more infected 
2AL

cM

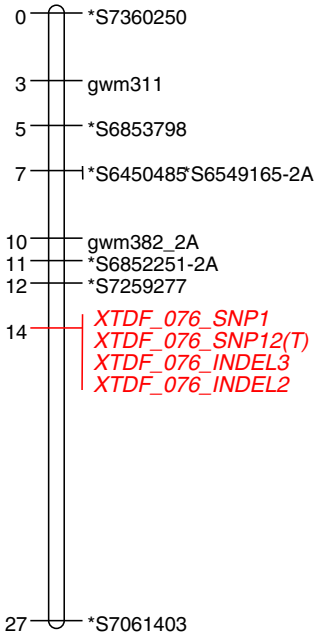

2DL

cM

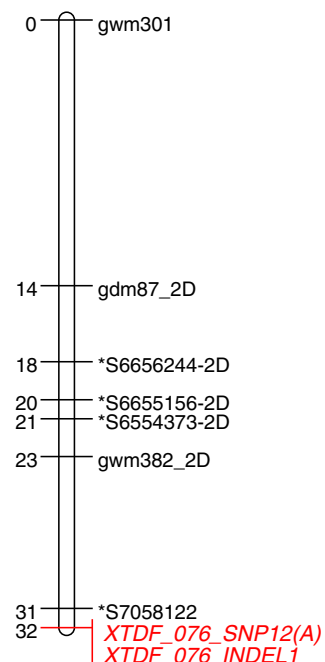

Fig. 1 Genetic map of chromosomes 2AL and 2DL of the Sngh3559//Capo/SVP72017 mapping population. The marker loci XTDF_076 were derived from a NPRl-like gene identified in the expression analysis described above. *AFLP markers were assigned to specific chromosomes with nulli-tetrasomic lines

spikelets than genotypes with the positive allele 2 of both homoeologous genes."

5. Under the heading "Discussion", the paragraph starting with "Allelic diversity in both homeologous genes...": The lines "None of the tested genotypes showed a combination of allele 1 of TDF_076_2A and allele 2 of TDF_076_2D" should read as "None of the tested genotypes showed a combination of allele 2 of TDF_076_2A and allele 1 of TDF_076_2D”. 